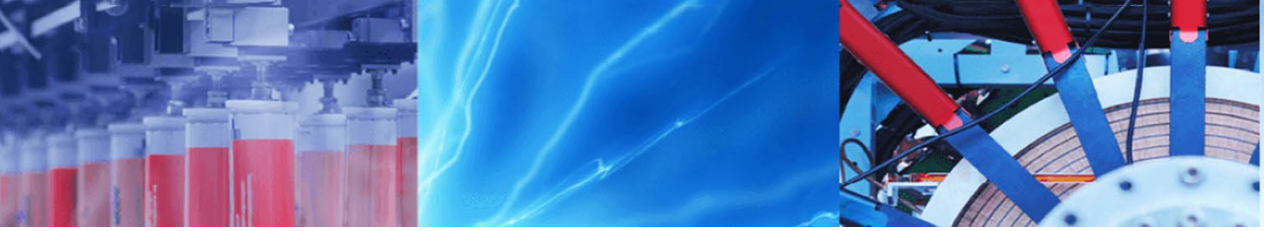

Research Article

\title{
A novel GPU based intrusion detection system using deep autoencoder with Fruitfly optimization
}

\author{
R. Sekhar ${ }^{1} \cdot$ K. Sasirekha ${ }^{2} \cdot$ P. S. Raja ${ }^{2} \cdot$ K. Thangavel ${ }^{2}$
}

Received: 6 October 2020 / Accepted: 15 April 2021

Published online: 01 May 2021

(C) The Author(s) 2021 OPEN

\begin{abstract}
Intrusion Detection Systems (IDSs) have received more attention to safeguarding the vital information in a network system of an organization. Generally, the hackers are easily entering into a secured network through loopholes and smart attacks. In such situation, predicting attacks from normal packets is tedious, much challenging, time consuming and highly technical. As a result, different algorithms with varying learning and training capacity have been explored in the literature. However, the existing Intrusion Detection methods could not meet the desired performance requirements. Hence, this work proposes a new Intrusion Detection technique using Deep Autoencoder with Fruitfly Optimization. Initially, missing values in the dataset have been imputed with the Fuzzy C-Means Rough Parameter (FCMRP) algorithm which handles the imprecision in datasets with the exploit of fuzzy and rough sets while preserving crucial information. Then, robust features are extracted from Autoencoder with multiple hidden layers. Finally, the obtained features are fed to Back Propagation Neural Network (BPN) to classify the attacks. Furthermore, the neurons in the hidden layers of Deep Autoencoder are optimized with population based Fruitfly Optimization algorithm. Experiments have been conducted on NSL_KDD and UNSW-NB15 dataset. The computational results of the proposed intrusion detection system using deep autoencoder with BPN are compared with Naive Bayes, Support Vector Machine (SVM), Radial Basis Function Network (RBFN), BPN, and Autoencoder with Softmax.
\end{abstract}

\section{Article Highlights}

- A hybridized model using Deep Autoencoder with Fruitfly Optimization is introduced to classify the attacks.

- Missing values have been imputed with the Fuzzy C-Means Rough Parameter method.

- The discriminate features are extracted using Deep Autoencoder with more hidden layers.

Keywords Autoencoder · Deep learning · Intrusion detection · Fruitfly · Back propagation neural network

\section{Introduction}

In a more digital and versatile world, it is very hard to safeguard private information with the aid of conventional security systems. Moreover, the number of attacks on computer networks has been increasing over the past few years. For detecting suspicious activity over the network with invasive behaviours such as vagueness, heterogeneity, convolution, and dynamic tendency, various methods are evolving continuously [1-3]. More recently, the accuracy of the intrusion detection and prevention system has been improved significantly with the

$\triangle$ R. Sekhar, rsekhar.bsf@nic.in; K. Sasirekha, ksasirekha@periyaruniversity.ac.in; P. S. Raja, psraja5@periyaruniversity.ac.in; K.Thangavel, drktvelu@periyaruniversity.ac.in | 'Disaster Recovery Centre, National Intelligence Grid, Ministry of Home Affairs, Bengaluru, India. ${ }^{2}$ Department of Computer Science, Periyar University, Salem, Tamilnadu, India.

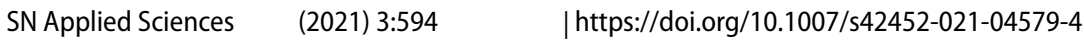


application of artificial intelligence based algorithms. In general, IDS are classified into signature, anomaly, host and network based systems. In particular, signature based detection is widely employed which draws rules sets to identify the patterns [4]. Normally, when IDS detect ongoing attack in the computer system, it raises alarm for taking action by the administrator $[5,6]$. The construction of efficient IDS in more crucial as the attacks on the network destroys huge volume of resources and paralyses entire network.

Typically, in the domain of artificial intelligence, the most dominant intelligent one is the deep learning models. The deep neural network has the ability to better learn latent representation automatically required for intrusion detection from raw data. Furthermore, the generalization ability of the deep neural network prevents the IDS from overfitting by generating relationships beyond immediate neighbours in the input [7]. One of the widely adopted architecture of deep neural network is stacked convolution autoencoder which trains the network in an unsupervised manner. Hence, in this work, the intrusion detection system is developed with the exploit of Deep Autoencoder to improve the performance of IDS using the adaptive and self-learning ability of the deep neural network. Moreover, Deep Autoencoder is straightforward and essential machine learning model for extracting more robust features from raw input. However, it suffers from the initialization of the number of hidden neurons. Among the swarm intelligence based optimization methods, it is observed that the Fruitfly algorithm is more widely adopted to fix the hyper parameters in many artificial intelligence applications [8]. One of the key features of Fruitfly Optimization algorithm is that it eliminates the local optimum problem and converges to global optimum quickly. As a result, the Fruitfly algorithm is implemented to optimize the neurons in the hidden layer of deep autoencoder.

In this paper, a Deep convolution Autoencoder with more hidden layers is used to extract more discriminate features from the input. Furthermore, the neurons of the hidden layers are optimized with Fruitfly Optimization algorithm. The missing values in the dataset have been imputed with the Fuzzy C-Means Rough Parameter algorithm to improve the performance.

The paper is organized as follows. In Sect. 2, the crucial intrusion detection methods are overviewed. In Sect. 3, the missing value imputation method FCMRP is presented. Section 4 presents the proposed intrusion detection method using Deep Autoencoder with Fruitfly algorithm. The computational results are presented under Sect. 5 . Finally, the conclusions and future research directions are given in Sect. 6.

\section{Related works}

More recently, the Deep Learning models play a significant role to handle more complex representation of the data. In [9], a hybridized method was introduced for handling missing values in the traffic dataset. Here, Fuzzy C-Means was employed to approximate the missing values. Further, the evolutionary genetic algorithm has been applied in order to optimize the hyper parameters such as centroids and membership functions in FCM algorithm.

Tian et al. [10] had developed an IDS using Radial Basic Function Network. The neural network can identify different intrusion activities fastly and efficiently by identifying the distinctive malicious characteristics information in the network. The computational results revealed that the developed intrusion detection system is viable and effectual to classify the attacks.

Mohammad et al. [11] classified network attacks in a system using conventional neural network. Here, two layer multi layer perceptron was developed with back propagation learning algorithm. The classification accuracy of $90.78 \%$ has been achieved.

Xiangmei et al. [12] had utilized neural network with Genetic Algorithm to construct an intrusion detection model. The computational results of the intrusion detection system with the developed method provide better accuracy.

Chualong et al. [13] had introduced Rough Neural Network based IDS for two class and multiclass attacks in the network. Experiments were conducted with varying the number of learning rate. The constructed classifier was compared with various benchmark classifiers such as J48, Random Forest and SVM to conclude the efficacy of the developed method. Alzubi et al. [14] had developed a feature selection algorithm named Binary Grey Wolf Optimization to obtain fine features from the dataset. Then, SVM was exploited to categorize the attacks. Experiments were conducted on KDD cup dataset and $99.22 \%$ accuracy was achieved. With the Binary Grey Wolf Optimization method, fourteen vital features were selected for classifying the network attacks. Qureshi et al. [15] introduced a deep neural network based IDS with self taught learning procedure. The features were extracted from the pre-trained network for classification. The experimental had been conducted on KDD cup dataset expound that the developed method improved by means of accuracy, and Receiver Operating Curve (ROC). In [16], the Genetic Algorithm was applied to choose the more optimal features from the intrusion dataset. The attacks were classified with Support Vector Machine and obtained a true-positive rate of 0.973 .

Rekha et al. [17] reviewed conventional machine learning algorithms such as Decision Tree, Random Forest, 
Apriori algorithm, Artificial Neural Network, etc. to detect IDS in a network. A comparative analysis with accuracy as a metric of various datasets has been presented. DrewekOssowicka et al. [18] compared various architectures of neural network utilized for creating an Intrusion Detection System. Liu et al. [19] implemented a deep Convolutional Neural Network model in order to detect network attack of an organization. Initially, the raw data was preprocessed to convert into two dimensional data. Further, more discriminate features were extracted using Convolutional Neural Network with Softmax classifier on KDD-CUP 99 and NSLKDD standard network intrusion detection dataset.

Most of the intrusion detection system discussed in the literature utilizes hand designed feature extractor for classification. Further, the hyperparameters are not optimized and the missing values are imputed randomly. Hence, these limitations are overcome in this research work.

\section{Pre-processing}

Generally, missing value imputation is one of the major challenging tasks that have to be handled in various machine learning applications. In this work, clustering based imputation has been utilized for imputing the missing values. Here, clustering is done with the dataset to obtain initial clusters. The missing values are imputed based on the cluster information.

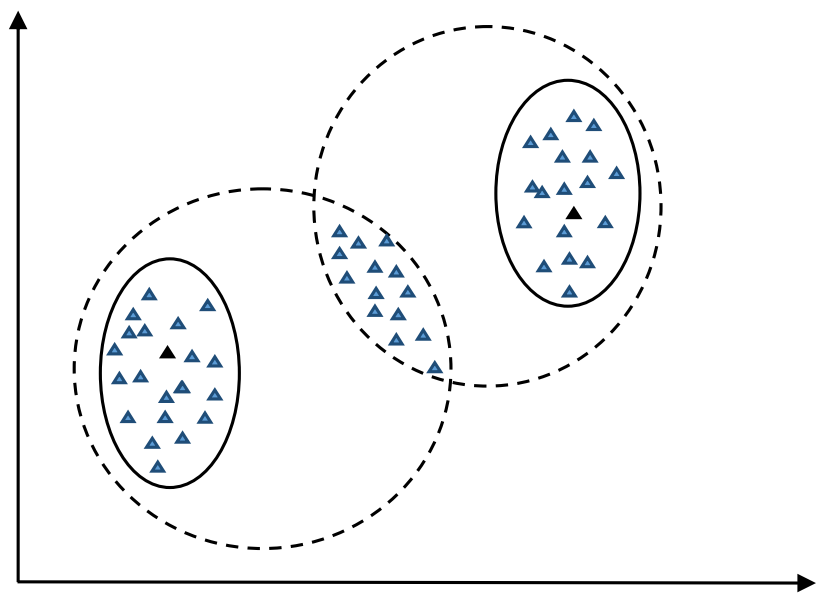

Fig. 1 Rough K-means centroid based imputation

\subsection{Rough K-means centroid based imputation method (RKMC)}

In RKMC, the centroid values obtained is used to impute the missing values exists in a particular dataset [20]. The complete procedure is given in algorithm 1 and Fig. 1 respectively. The centriod has been measured by

$m_{k}=w_{I} \sum_{x_{i} \in \underline{C_{k}}} \frac{x_{i}}{\left|\underline{C_{k}}\right|}+w_{u} \sum_{x_{i} \in \overline{C_{k}}} \frac{x_{i}}{\left|\overline{C_{k}}\right|}$ 
Algorithm 1 : RKMC algorithm

Input $\quad$ : Dataset $X$ of $\mathrm{N}$ objects with missing attribute values, threshold, $w_{l}, w_{u}$ and number of clusters $K$

Output : Objects without missing values (Imputed Dataset)

Step 1: Given dataset of $\mathrm{N}$ objects $X=\left\{x_{i} \mid i=1,2, \ldots, N\right\}, C X \subset \mathrm{X}, M X \subset \mathrm{X}$, where $\mathrm{N}$ is the number of objects. $C X$ holds object with complete data and $M X$ holds object with incomplete data

Step 2: Initialize the number of clusters K.

Step 3: RKMC is applied on CX. The centroid measure for Rough K-Means clustering algorithm is computed by

$$
m_{k}=w_{l} \sum_{x_{i} \in \underline{C_{k}}} \frac{x_{i}}{\left|\underline{C_{k}}\right|}+w_{u} \sum_{x_{i} \in C_{k}} \frac{x_{i}}{\left|\overline{C_{k}}\right|}
$$

Step 4: The object $m x_{\mathrm{i}}$ missing column value is replaced by corresponding $\mathrm{m}_{\mathrm{k}}$ column value and find the minimum distance between $m x_{\mathrm{i}}$ and $\mathrm{m}_{\mathrm{k}}$ here $\mathrm{k}=1,2, \ldots, \mathrm{K}$.

$$
d_{i, k}^{m i n}=\operatorname{dist}\left(m x_{i}, m_{k}\right)=\min _{k=1, \ldots, K} \operatorname{dist}\left(m x_{i}, m_{k}\right)
$$

where $\operatorname{dist}\left(m x_{i}, m_{k}\right)$ computes the distance between $m x_{i}$ and $m_{k}$.

Step 5: Replace the $m x_{i}$ missing attribute values with corresponding $\mathrm{m}_{\mathrm{h}}$ attribute values and place it in the $\mathrm{C}_{\mathrm{h}}$ cluster.

Step 6: Repeat step 4 to 5 for all instances in $M X$.

Step 7: Stop.

\subsection{Fuzzy C-means rough parameter based imputation method}

The FCMRP algorithm consists of three steps. Initially, FCM clustering algorithm is applied to the objects in order to group the data into clusters. Then, find the centroid and nearest approximation in the cluster for each missing object. Finally, the missing values exists in each incomplete object is imputed with the lower and upper approximation of the cluster [21]. The centroid of FCM is computed by

$m_{k}=\frac{\sum_{\mathrm{i}=1}^{\mathrm{N}} \mathrm{u}_{\mathrm{ij}}^{\mathrm{v}} \cdot x_{\mathrm{i}}}{\sum_{\mathrm{i}=1}^{N} \mathrm{u}_{\mathrm{ij}}^{\mathrm{v}}}$
In FCMRP, the rough clustering applied to the clusters attained with FCM method. Here, the imputation is carried out in lower and upper approximation separately. The incomplete data in each object is imputed by

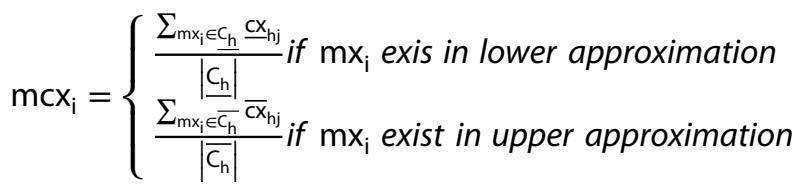

Algorithm 2 shows the steps involved in FCMRP to handle missing values in a dataset. Figure 2 depicts the FCMRP with its clusters $\mathrm{C}_{1}, \mathrm{C}_{2}, \mathrm{C}_{3}, \mathrm{RC}_{11}$ and $\mathrm{RC}_{12}$. 
Algorithm 2 : FCMRP algorithm

Input : Dataset $X$ of $\mathrm{N}$ objects with missing attribute values, membership matrix and number of clusters $K$

Output : Objects without missing values (Complete Dataset)

Step 1: Given dataset of N objects $X=\left\{x_{1}, x_{2}, \ldots, x_{N}\right\}, C X \subset \mathrm{X}, M X \subset \mathrm{X}$, where $\mathrm{N}$ is the number of objects, $C X$ contain objects with complete data and $M X$ contains objects with incomplete attribute values.

Step 2: Initialize the number of clusters K.

Step 3: FCM is applied to $C X$ and the centroid is obtained as

$$
m_{k}=\frac{\sum_{\mathrm{i}=1}^{\mathrm{N}} \mathrm{u}_{\mathrm{ij}}^{\mathrm{v}} \cdot x_{\mathrm{i}}}{\sum_{\mathrm{i}=1}^{N} \mathrm{u}_{\mathrm{ij}}^{\mathrm{v}}}
$$

Step 4: Apply Rough K-Means to each clusters obtained by FCM.

Step 5: The missing object $m x_{\mathrm{i}}$ is replaced with corresponding $\mathrm{m}_{\mathrm{k}}$ column value and find the least distance between $m x_{\mathrm{i}}$ and centroid by

$$
d_{i, k}^{\min }=\operatorname{dist}\left(m x_{i}, m_{k}\right)=\min _{k=1, \ldots, K} \operatorname{dist}\left(m x_{i}, m_{k}\right)
$$

where $\operatorname{dist}\left(m x_{i}, m_{k}\right)$ computes the distance between $m x_{i}$ and $m_{k}$

Step 6: Find the nearest approximation to the missing object $m x_{i}$.

Step 7: In Rough clustering, find the closest approximation to the cluster $\mathrm{C}_{\mathrm{h}}$ and use that information to impute the missing

values using

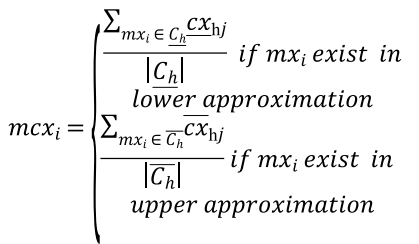

Step 8: The missing value of $m x_{i}$ is replaced with the corresponding column value of $m c x_{i}$ Column value.

Step 9: Repeat step 5 to 8 for all objects in $M X$

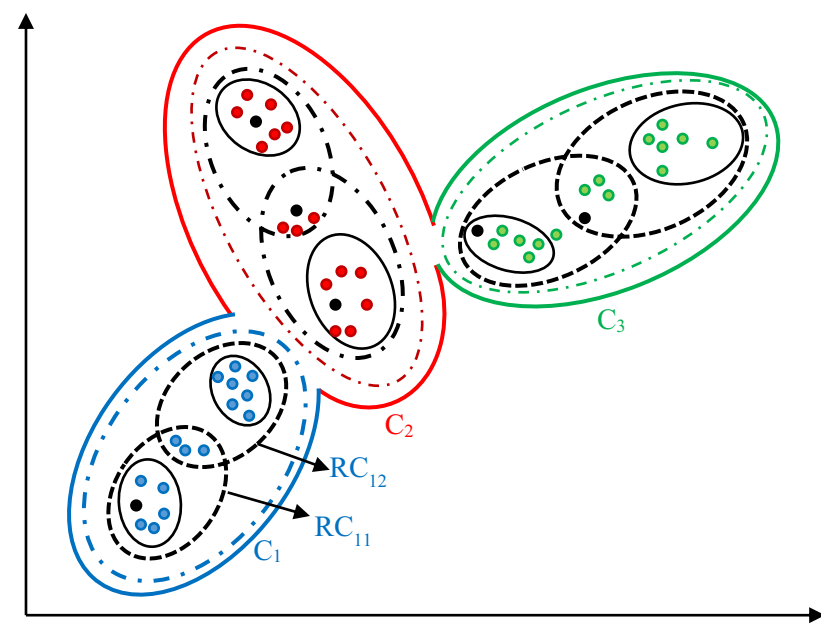

Fig. 2 FCMRP based imputation method

\section{Methodology}

The proposed intrusion detection system by Deep Autoencoder with Fruitfly Optimization and BPN is discussed in this section.

\subsection{Deep autoencoder}

Autoencoder is a special kind of multilayer perceptron where the number of neurons in the input and output layer is same. A deep autoencoder will be constructed by stacking several autoencoders as in Deep Belief Networks (DBNs) [22-24]. It is used to learn more complex representations from the raw input for further analysis. 


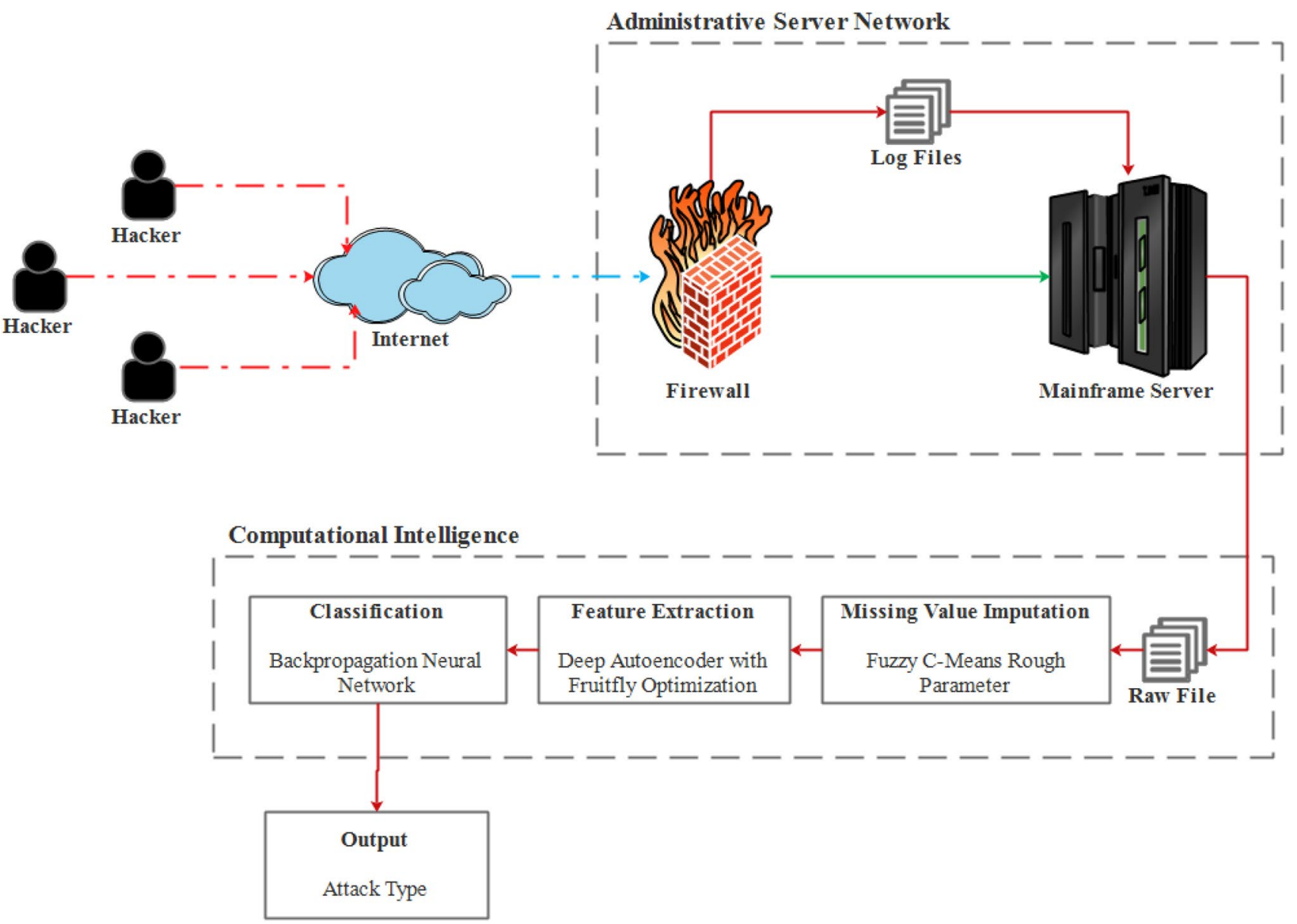

Fig. 3 Overview of the proposed IDS

The architecture of autoencoder includes two parts, encoder and decoder which are trained at each successive layer. While constructing a deep autoencoder, each layer receives its input from previous layer. In particularly, the autoencoder is trained to convert the raw input into some latent or more abstract representation and the output is reconstructed from that compressed representation [25-29]. The encoder receives the raw data as input $I(x) \in R^{d}$ and maps into a latent representation $H(x) \in R^{\prime}$ with the function as,

$H(x)=\sigma(W * I(x)+b)$

Here, sigmoid is used as the learning function. W and $\mathrm{b}$ are the weight and bias respectively. The decoder part reconstructs the input back at the output layer with the function as given in (5).
$R(x)=\sigma\left(W^{\prime} * H(x)+b^{\prime}\right)$

where $R(x)$ is the predicted output of the input $I(x)$ from the latent representation $H(x)$. The weights of the autoencoders are optimized to reduce the reconstruction error of the network. The reconstruction error is computed as,

$L(I(x), R(x))=\|I(x)-R(x)\|^{2}$

Further, the cross-entropy measure is used to compute the reconstruction error for binary values using (7).

$L(I(x), R(x))=-\sum_{k=1}^{d}\left[I(x)_{k} \log R(x)_{k}+\left(1 /(x)_{k}\right) \log \left(1-R(x) r_{k}\right)\right]$ 
Fig. 4 Feature extraction using deep autoencoder

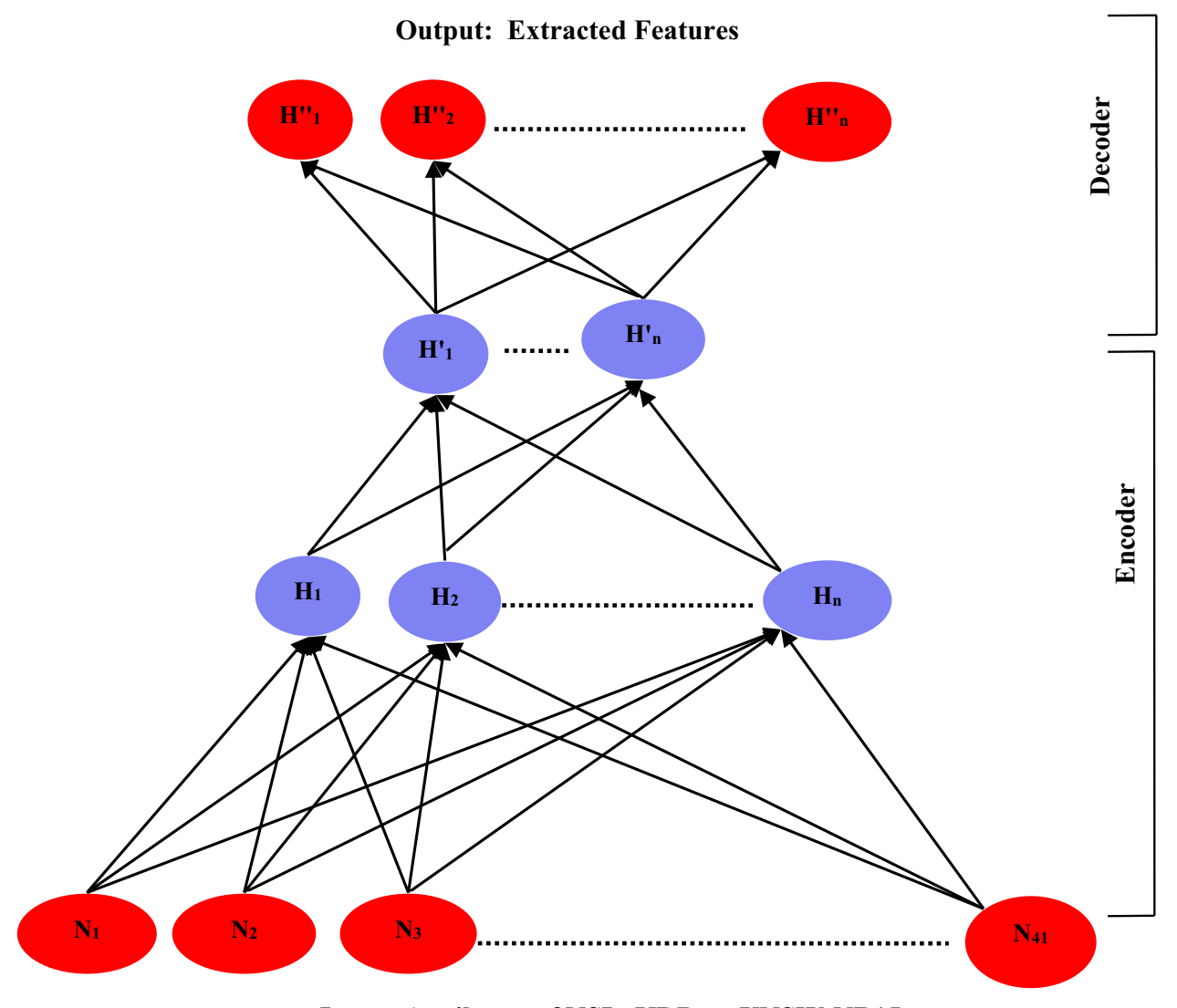

Input: Attributes of NSL_KDD or UNSW-NB15

Fig. 5 Architecture of BPN for attack classification

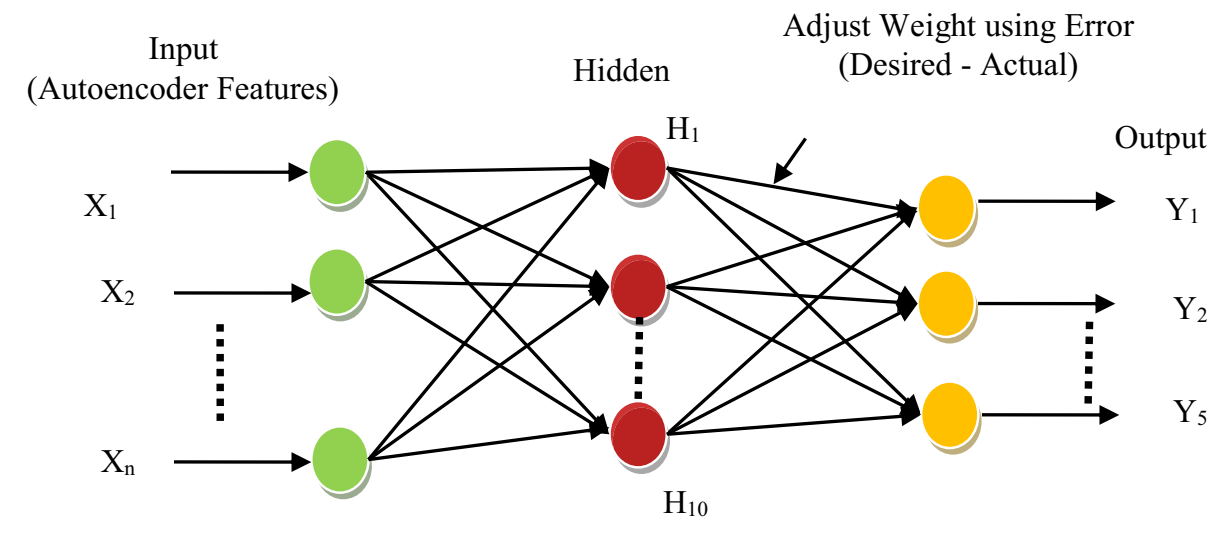

$1 \times$ Supermicro 1 U Chassis with 1400 W High Efficiency power supply Platinum Level

$1 \times$ Single socket R3 (LGA 2011) Intel Xeon Processor E5-2600 v4 +/v3 and E5-1600 v4 +/v3 family

$1 \times$ Intel Broadwell E5-2620 v4 2.1 GHz 8 Core 20 MB 8 GT/s QPI

$32 \mathrm{~GB}(2 \times 16 \mathrm{~GB})$ DDR4 ECC-Registered $2400 \mathrm{MHz}$ Memory

$1 \times$ Enterprises class 1 TB SATA 7.2 K RPM 2.5" HDD

$1 \times$ Nvidia Tesla P100 GPU, 12 GB 
Table 2 Quantitative performance on UNSW-NB15 dataset

\begin{tabular}{|c|c|c|c|c|c|c|}
\hline \multirow[t]{2}{*}{ Dataset } & \multirow{2}{*}{$\begin{array}{l}\text { Missing } \\
\text { combina- } \\
\text { tions (\%) }\end{array}$} & \multicolumn{2}{|c|}{$\begin{array}{l}\text { Actual posi- } \\
\text { tion }\end{array}$} & \multirow[t]{2}{*}{ Actual value } & \multicolumn{2}{|l|}{ Imputed value } \\
\hline & & Row & Column & & $\begin{array}{l}\text { Rough K-means } \\
\text { centroid based impu- } \\
\text { tation }\end{array}$ & $\begin{array}{l}\text { Fuzzy rough param- } \\
\text { eter based imputa- } \\
\text { tion }\end{array}$ \\
\hline \multirow[t]{25}{*}{ UNSW-NB15 } & \multirow[t]{5}{*}{5} & 1298 & 3 & 1 & 1 & 1 \\
\hline & & 1340 & 3 & 0 & 1 & 0 \\
\hline & & 1354 & 3 & 0 & 0 & 0 \\
\hline & & 1396 & 3 & 0 & 0 & 0 \\
\hline & & 1457 & 3 & 0 & 1 & 0 \\
\hline & \multirow[t]{5}{*}{10} & 123 & 3 & 0 & 0 & 0 \\
\hline & & 124 & 3 & 0 & 0 & 0 \\
\hline & & 191 & 3 & 0 & 0 & 0 \\
\hline & & 215 & 3 & 5 & 1 & 5 \\
\hline & & 216 & 3 & 1 & 1 & 1 \\
\hline & \multirow[t]{5}{*}{15} & 522 & 3 & 0 & 1 & 1 \\
\hline & & 526 & 3 & 0 & 0 & 0 \\
\hline & & 559 & 3 & 0 & 0 & 0 \\
\hline & & 609 & 3 & 0 & 0 & 0 \\
\hline & & 630 & 3 & 0 & 1 & 0 \\
\hline & \multirow[t]{5}{*}{20} & 7 & 3 & 0 & 1 & 0 \\
\hline & & 11 & 3 & 0 & 1 & 0 \\
\hline & & 46 & 3 & 9 & 1 & 1 \\
\hline & & 64 & 3 & 0 & 0 & 0 \\
\hline & & 71 & 3 & 0 & 0 & 0 \\
\hline & \multirow[t]{5}{*}{25} & 8 & 3 & 0 & 1 & 0 \\
\hline & & 17 & 3 & 0 & 1 & 0 \\
\hline & & 19 & 3 & 1 & 0 & 1 \\
\hline & & 34 & 3 & 0 & 1 & 0 \\
\hline & & 43 & 3 & 0 & 0 & 0 \\
\hline
\end{tabular}

Table 3 RMSE and MAE on UNSW-NB15 dataset

\begin{tabular}{|c|c|c|c|c|c|c|c|}
\hline \multirow[t]{2}{*}{ Dataset } & \multirow[t]{2}{*}{ Clustering algorithm } & \multirow{2}{*}{$\begin{array}{l}\text { Evaluation } \\
\text { measures }\end{array}$} & \multicolumn{5}{|c|}{ Missing combinations } \\
\hline & & & $5 \%$ & $10 \%$ & $15 \%$ & $20 \%$ & $25 \%$ \\
\hline \multirow[t]{4}{*}{ UNSW-NB15 } & \multirow[t]{2}{*}{ Rough K-means centroid based imputation } & RMSE & 7.1038 & 5.0140 & 4.4368 & 3.4156 & 3.4039 \\
\hline & & MAE & 2.1676 & 1.1165 & 0.9951 & 0.8871 & 0.8057 \\
\hline & \multirow[t]{2}{*}{ Fuzzy rough parameter based imputation } & RMSE & 4.5378 & 3.0600 & 4.1094 & 2.7115 & 2.1898 \\
\hline & & MAE & 1.4111 & 0.8484 & 0.8697 & 0.7851 & 0.7171 \\
\hline
\end{tabular}


Table 4 Performance of RKMC on UNSW-NB15 dataset

\begin{tabular}{|c|c|c|c|c|c|c|}
\hline \multirow[t]{2}{*}{ Object ID } & \multicolumn{4}{|c|}{ Distance between object and cluster centroid } & \multirow{2}{*}{$\begin{array}{l}\text { Minimum } \\
\text { distance }\end{array}$} & \multirow{2}{*}{$\begin{array}{l}\text { Minimum } \\
\text { distance } \\
\text { cluster }\end{array}$} \\
\hline & Cluster 1 & Cluster 2 & Cluster 3 & Cluster K & & \\
\hline 1 & 126.29 & 253.20 & 267.09 & 329.91 & 46.60 & 9 \\
\hline 2 & 87.69 & 343.61 & 389.21 & 435.10 & 87.69 & 1 \\
\hline 3 & 385.59 & 228.18 & 305.67 & 540.28 & 228.18 & 2 \\
\hline 4 & 463.13 & 133.13 & 316.30 & 314.77 & 214.77 & 4 \\
\hline 5 & 232.86 & 167.97 & 354.50 & 231.70 & 154.70 & 13 \\
\hline 6 & 187.69 & 343.61 & 389.21 & 435.10 & 187.69 & 1 \\
\hline 7 & 447.47 & 212.18 & 179.02 & 152.10 & 61.01 & 10 \\
\hline 8 & 187.69 & 343.61 & 389.21 & 435.10 & 187.69 & 1 \\
\hline 9 & 242.00 & 330.02 & 167.93 & 371.25 & 149.30 & 12 \\
\hline 10 & 187.69 & 343.61 & 389.21 & 435.10 & 187.69 & 1 \\
\hline
\end{tabular}

Table 5 Performance of FCMRP on UNSW-NB15 dataset

Table 6 Fruitfly parameters

\begin{tabular}{|c|c|c|c|c|c|c|}
\hline \multirow[t]{2}{*}{ Object ID } & \multicolumn{4}{|c|}{ Distance between object and cluster centroid } & \multirow{2}{*}{$\begin{array}{l}\text { Minimum } \\
\text { distance }\end{array}$} & \multirow{2}{*}{$\begin{array}{l}\text { Minimum } \\
\text { distance } \\
\text { cluster }\end{array}$} \\
\hline & Cluster 1 & Cluster 2 & Cluster 3 & Cluster K & & \\
\hline 1 & 126.38 & 337.38 & 304.48 & 156.33 & 56.33 & 4 \\
\hline 2 & 108.95 & 443.32 & 428.40 & 897.06 & 108.95 & 1 \\
\hline 3 & 385.68 & 628.76 & 308.31 & 307.65 & 234.70 & 12 \\
\hline 4 & 463.22 & 102.26 & 307.58 & 381.78 & 102.26 & 2 \\
\hline 5 & 232.93 & 240.55 & 377.38 & 177.91 & 154.41 & 6 \\
\hline 6 & 108.95 & 443.32 & 428.40 & 897.06 & 108.95 & 1 \\
\hline 7 & 447.57 & 149.71 & 161.03 & 359.02 & 69.75 & 8 \\
\hline 8 & 17.89 & 443.32 & 428.40 & 897.06 & 17.89 & 1 \\
\hline 9 & 242.08 & 376.27 & 210.44 & 179.52 & 149.65 & 13 \\
\hline 10 & 78.95 & 443.32 & 428.40 & 897.06 & 78.95 & 1 \\
\hline
\end{tabular}

\begin{tabular}{ll}
\hline Parameter & Value \\
\hline Swarm size & 50 \\
Iterations & 30 \\
Distribution radius & $(0,1)$ \\
\hline
\end{tabular}


Table 7 Confusion matrix of autoencoder with softmax classifier on NSL_KDD dataset

\begin{tabular}{|c|c|c|c|c|c|c|}
\hline & $\begin{array}{c}5931 \\
48.8 \%\end{array}$ & $\begin{array}{c}3 \\
0.0 \%\end{array}$ & $\begin{array}{c}146 \\
1.2 \%\end{array}$ & $\begin{array}{c}8 \\
0.1 \%\end{array}$ & $\begin{array}{c}11 \\
0.1 \%\end{array}$ & $\begin{array}{l}97.2 \% \\
2.8 \%\end{array}$ \\
\hline & $\begin{array}{c}0 \\
0.0 \%\end{array}$ & $\begin{array}{c}1 \\
0.0 \%\end{array}$ & $\begin{array}{c}0 \\
0.0 \% \\
\end{array}$ & $\begin{array}{c}0 \\
0.0 \%\end{array}$ & $\begin{array}{c}0 \\
0.0 \%\end{array}$ & $\begin{array}{l}100 \% \\
0.0 \%\end{array}$ \\
\hline & $\begin{array}{c}30 \\
0.2 \%\end{array}$ & $\begin{array}{c}10 \\
0.1 \% \\
\end{array}$ & $\begin{array}{c}150 \\
1.2 \% \\
\end{array}$ & $\begin{array}{c}7 \\
0.1 \% \\
\end{array}$ & $\begin{array}{c}60 \\
0.5 \% \\
\end{array}$ & $\begin{array}{l}58.4 \% \\
41.6 \% \\
\end{array}$ \\
\hline & $\begin{array}{c}20 \\
0.2 \% \\
\end{array}$ & \begin{tabular}{|c|}
30 \\
$0.2 \%$ \\
\end{tabular} & $\begin{array}{c}0 \\
0.0 \%\end{array}$ & $\begin{array}{l}478 \\
3.9 \%\end{array}$ & $\begin{array}{c}2 \\
0.0 \%\end{array}$ & $9.8 \%$ \\
\hline & $\begin{array}{c}56 \\
0.5 \%\end{array}$ & $\begin{array}{c}215 \\
1.8 \% \\
\end{array}$ & $\begin{array}{c}422 \\
3.5 \% \\
\end{array}$ & $\begin{array}{c}5 \\
0.0 \% \\
\end{array}$ & $\begin{array}{l}4563 \\
37.6 \% \\
\end{array}$ & \\
\hline & $\begin{array}{l}98.2 \% \\
1.8 \%\end{array}$ & $\begin{array}{l}0.4 \% \\
99.6 \%\end{array}$ & $\begin{array}{l}20.9 \% \\
79.1 \%\end{array}$ & $\begin{array}{l}96.0 \% \\
4.0 \%\end{array}$ & $\begin{array}{l}98.4 \% \\
1.6 \%\end{array}$ & $\begin{array}{l}91.6 \% \\
8.4 \%\end{array}$ \\
\hline
\end{tabular}
1
2
3
5

Table 8 Confusion matrix of autoencoder with BPN on NSL_KDD dataset

\begin{tabular}{|c|c|c|c|c|c|c|}
\hline 1 & $\begin{array}{c}4476 \\
49.1 \% \\
\end{array}$ & $\begin{array}{c}0 \\
0.0 \%\end{array}$ & $\begin{array}{c}41 \\
0.5 \%\end{array}$ & $\begin{array}{c}2 \\
0.0 \%\end{array}$ & $\begin{array}{c}11 \\
0.1 \%\end{array}$ & $\begin{array}{l}98.8 \% \\
1.2 \% \\
\end{array}$ \\
\hline 2 & $\begin{array}{c}0 \\
0.0 \%\end{array}$ & $\begin{array}{c}12 \\
0.1 \%\end{array}$ & $\begin{array}{c}1 \\
0.0 \%\end{array}$ & $\begin{array}{c}3 \\
0.0 \%\end{array}$ & $\begin{array}{c}0 \\
0.0 \%\end{array}$ & $\begin{array}{l}75.0 \% \\
25.0 \%\end{array}$ \\
\hline 3 & \begin{tabular}{|c|}
10 \\
$0.1 \%$ \\
\end{tabular} & \begin{tabular}{|c|}
8 \\
$0.1 \%$ \\
\end{tabular} & $\begin{array}{c}260 \\
2.9 \% \\
\end{array}$ & \begin{tabular}{|c|}
3 \\
$0.0 \%$ \\
\end{tabular} & \begin{tabular}{|c|}
35 \\
$0.4 \%$ \\
\end{tabular} & \\
\hline 4 & $\begin{array}{c}10 \\
0.1 \% \\
\end{array}$ & \begin{tabular}{|c|}
4 \\
$0.0 \%$ \\
\end{tabular} & $\begin{array}{c}1 \\
0.0 \% \\
\end{array}$ & \begin{tabular}{c|}
355 \\
$3.9 \%$ \\
\end{tabular} & $\begin{array}{c}10 \\
0.1 \% \\
\end{array}$ & $6.6 \%$ \\
\hline 5 & \begin{tabular}{|c|}
41 \\
$0.5 \%$ \\
\end{tabular} & $\begin{array}{c}201 \\
2.2 \% \\
\end{array}$ & \begin{tabular}{|c|}
271 \\
$3.0 \%$ \\
\end{tabular} & \begin{tabular}{|c|}
9 \\
$0.1 \%$ \\
\end{tabular} & $\begin{array}{c}3347 \\
36.7 \% \\
\end{array}$ & $\begin{array}{l}86.5 \% \\
13.5 \% \\
\end{array}$ \\
\hline & $\begin{array}{l}98.7 \% \\
1.3 \%\end{array}$ & $\begin{array}{l}5.3 \% \\
94.7 \%\end{array}$ & $\begin{array}{l}45.3 \% \\
54.7 \%\end{array}$ & $\begin{array}{l}95.4 \% \\
4.6 \%\end{array}$ & $\begin{array}{c}98.4 \% \\
1.6 \%\end{array}$ & $\begin{array}{l}92.7 \% \\
7.3 \%\end{array}$ \\
\hline & 1 & 2 & 3 & 4 & 5 & \\
\hline
\end{tabular}

Table 9 Confusion matrix for fruitfly-autoencoder with BPN on NSL_KDD dataset

\begin{tabular}{|c|c|c|c|c|c|c|}
\hline 1 & $\begin{array}{c}4524 \\
49.7 \%\end{array}$ & $\begin{array}{c}1 \\
0.0 \%\end{array}$ & $\begin{array}{c}45 \\
0.5 \%\end{array}$ & $\begin{array}{c}1 \\
0.0 \%\end{array}$ & $\begin{array}{c}11 \\
0.1 \%\end{array}$ & $\begin{array}{l}98.7 \% \\
1.3 \%\end{array}$ \\
\hline 2 & $\begin{array}{c}0 \\
0.0 \%\end{array}$ & $\begin{array}{c}17 \\
0.2 \%\end{array}$ & $\begin{array}{c}7 \\
0.1 \%\end{array}$ & $\begin{array}{c}3 \\
0.0 \%\end{array}$ & $\begin{array}{c}1 \\
0.0 \%\end{array}$ & $\begin{array}{l}60.7 \% \\
39.3 \%\end{array}$ \\
\hline 3 & $\begin{array}{c}7 \\
0.1 \%\end{array}$ & $\begin{array}{c}4 \\
0.0 \% \\
\end{array}$ & $\begin{array}{c}239 \\
2.6 \%\end{array}$ & $\begin{array}{c}2 \\
0.0 \% \\
\end{array}$ & $\begin{array}{c}26 \\
0.3 \% \\
\end{array}$ & $\begin{array}{l}86.0 \% \\
14.0 \% \\
\end{array}$ \\
\hline 4 & $\begin{array}{c}7 \\
0.1 \%\end{array}$ & $\begin{array}{c}0 \\
0.0 \%\end{array}$ & $\begin{array}{c}0 \\
0.0 \%\end{array}$ & $\begin{array}{c}351 \\
3.9 \%\end{array}$ & $\begin{array}{c}5 \\
0.1 \%\end{array}$ & $3.3 \%$ \\
\hline 5 & $\begin{array}{c}41 \\
0.5 \%\end{array}$ & $\begin{array}{c}152 \\
1.7 \%\end{array}$ & $\begin{array}{c}229 \\
2.5 \%\end{array}$ & $\begin{array}{c}7 \\
0.1 \%\end{array}$ & $\begin{array}{r}3431 \\
37.7 \%\end{array}$ & $\begin{array}{l}88.9 \% \\
11.1 \%\end{array}$ \\
\hline & $\begin{array}{l}98.8 \% \\
1.2 \%\end{array}$ & $\begin{array}{l}9.8 \% \\
90.2 \%\end{array}$ & $\begin{array}{l}46.0 \% \\
54.0 \%\end{array}$ & $\begin{array}{c}96.4 \% \\
3.6 \%\end{array}$ & $\begin{array}{c}98.8 \% \\
1.2 \%\end{array}$ & $\begin{array}{l}94.0 \% \\
6.0 \%\end{array}$ \\
\hline
\end{tabular}

$\begin{array}{lllll}1 & 2 & 3 & 4 & 5\end{array}$

\subsection{Fruitfly optimization}

The Fruitfly is a population based optimization method introduced by Pan in 2012 [30]. This algorithm is stimulated by the foraging behavior of FruitFlies. More specifically, it has the capability to smell the food source from a very long distance. Hence, the Fruitfly algorithm has been adopted to optimize the parameters in various real-time applications recently [31, 32]. In this work, the Fruitfly is adopted to optimize the neurons of hidden layers in deep autoencoder. 
Table 10 Comparative analysis of proposed intrusion detection system with existing benchmark classifiers on NSL_ KDD dataset

\begin{tabular}{llllll}
\hline Classification algorithm & Precision & Recall & F-measure & Accuracy & Error rate \\
\hline Naïve Bayes & 70.10 & 71.10 & 70.40 & 71.14 & 28.86 \\
SVM & 87.00 & 87.20 & 86.90 & 87.15 & 12.85 \\
RBFN & 87.70 & 89.90 & 87.60 & 87.86 & 12.14 \\
BPN & 89.35 & 90.60 & 89.97 & 91.00 & 09.00 \\
Autoencoder-softmax & 90.05 & 91.00 & 90.52 & 91.60 & 08.40 \\
Autoencoder-BPN & 91.35 & 92.15 & 91.74 & 92.70 & 07.30 \\
Fruitfly-autoencoder-BPN & 92.90 & 93.60 & 93.24 & 94.00 & 06.00 \\
\hline
\end{tabular}

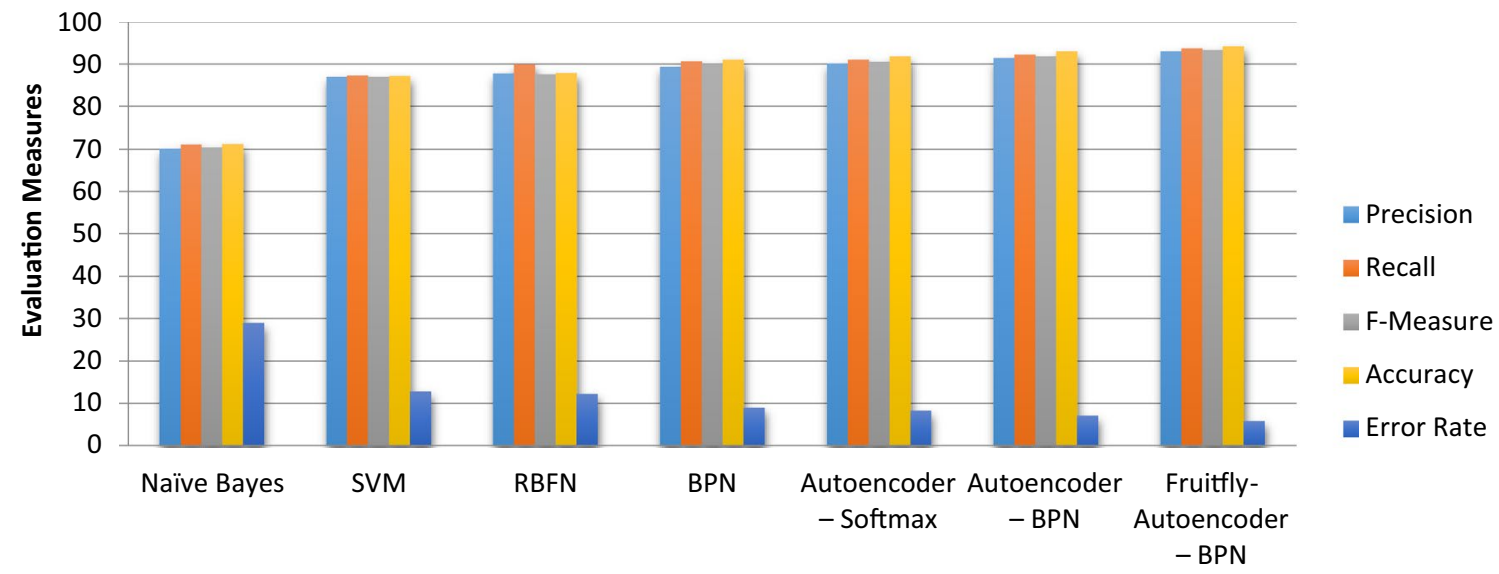

Classifiers

Fig. 6 Relative quantitative measures for intrusion detection system on NSL_KDD dataset

Table 11 Confusion matrix of autoencoder with softmax classifier on UNSW-NB15 dataset

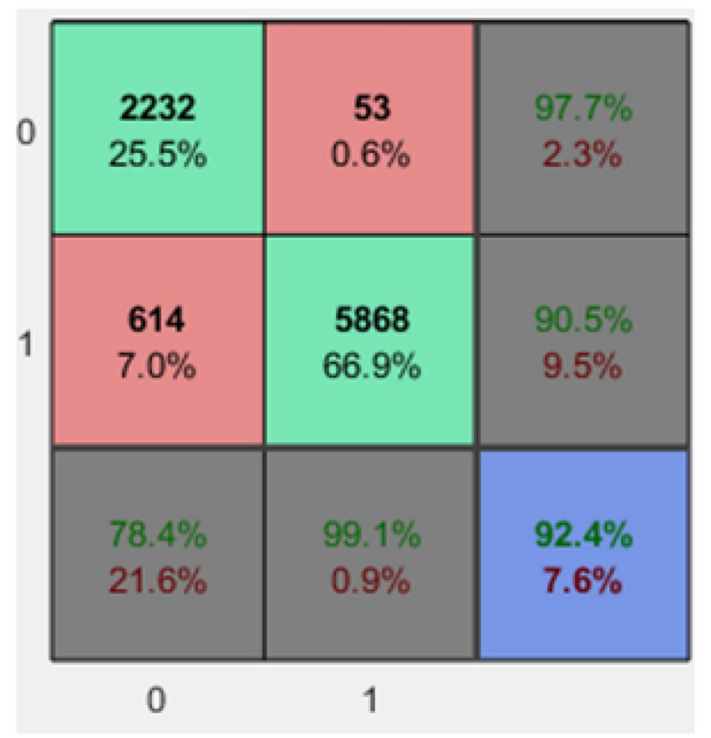

Table 12 Confusion matrix of autoencoder with BPN on UNSWNB15 dataset

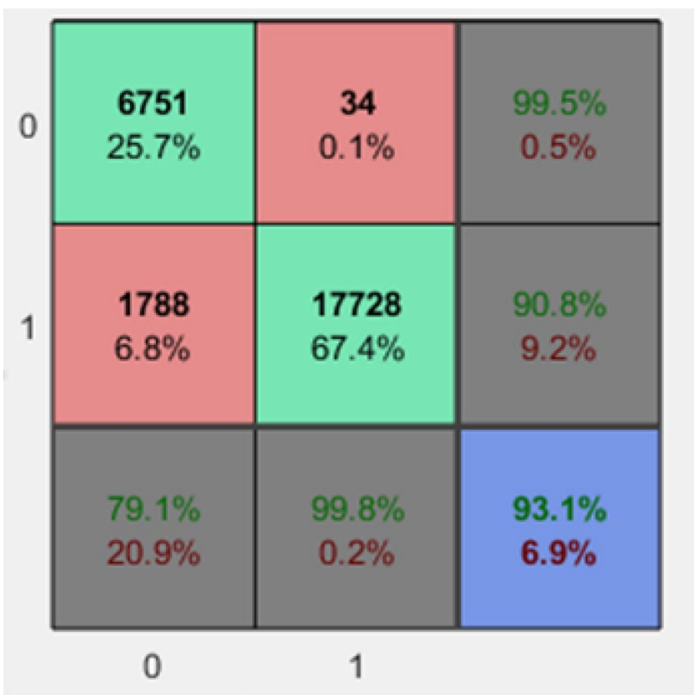


Table 13 Confusion matrix of fruitfly-autoencoder with BPN on UNSW-NB15 dataset

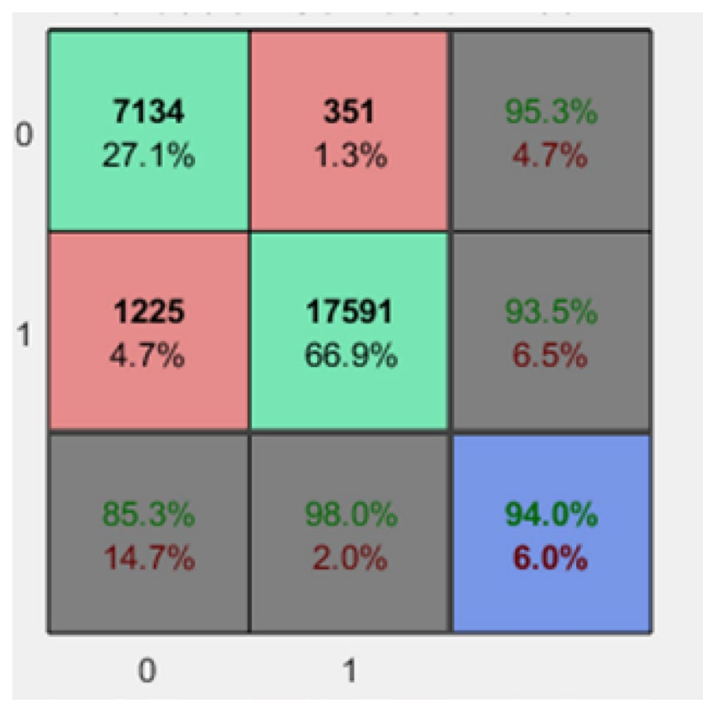

\subsection{Proposed intrusion detection technique}

The Deep Autoencoder is trained with multiple hidden layers to extract the optimal features from raw data. Here, Fruitfly algorithm is implemented to optimize the hidden neurons of deep autoencoder while training. Furthermore, to classify the attacks into Denial of Service (DoS), User-toRoot (U2R), Remote-to-Locals (R2L), Probe, and Normal, the backpropagation neural network is exploited. The complete procedure of the proposed IDS is given in Fig. 3 . The procedure for learning and stacking several layers of autoencoders is presented in Fig. 4. The classification of attacks in a network system is presented in algorithm 3.
Table 14 Comparative analysis of proposed intrusion detection system with existing benchmark classifiers on UNSW-NB15 dataset

\begin{tabular}{|c|c|c|c|c|c|}
\hline Classification algorithm & Precision & Recall & F-measure & Accuracy & Error rate \\
\hline Naïve Bayes & 86.20 & 87.30 & 87.30 & 89.31 & 10.69 \\
\hline SVM & 88.20 & 89.50 & 88.85 & 88.20 & 11.80 \\
\hline RBFN & 92.00 & 88.45 & 90.19 & 89.60 & 10.40 \\
\hline BPN & 90.50 & 90.10 & 90.30 & 90.30 & 09.70 \\
\hline Autoencoder-Softmax & 90.35 & 91.15 & 90.74 & 92.40 & 07.60 \\
\hline Autoencoder-BPN & 92.50 & 92.80 & 92.65 & 93.10 & 06.90 \\
\hline Fruitfly-Autoencoder-BPN & 92.50 & 93.20 & 92.84 & 94.00 & 06.00 \\
\hline
\end{tabular}

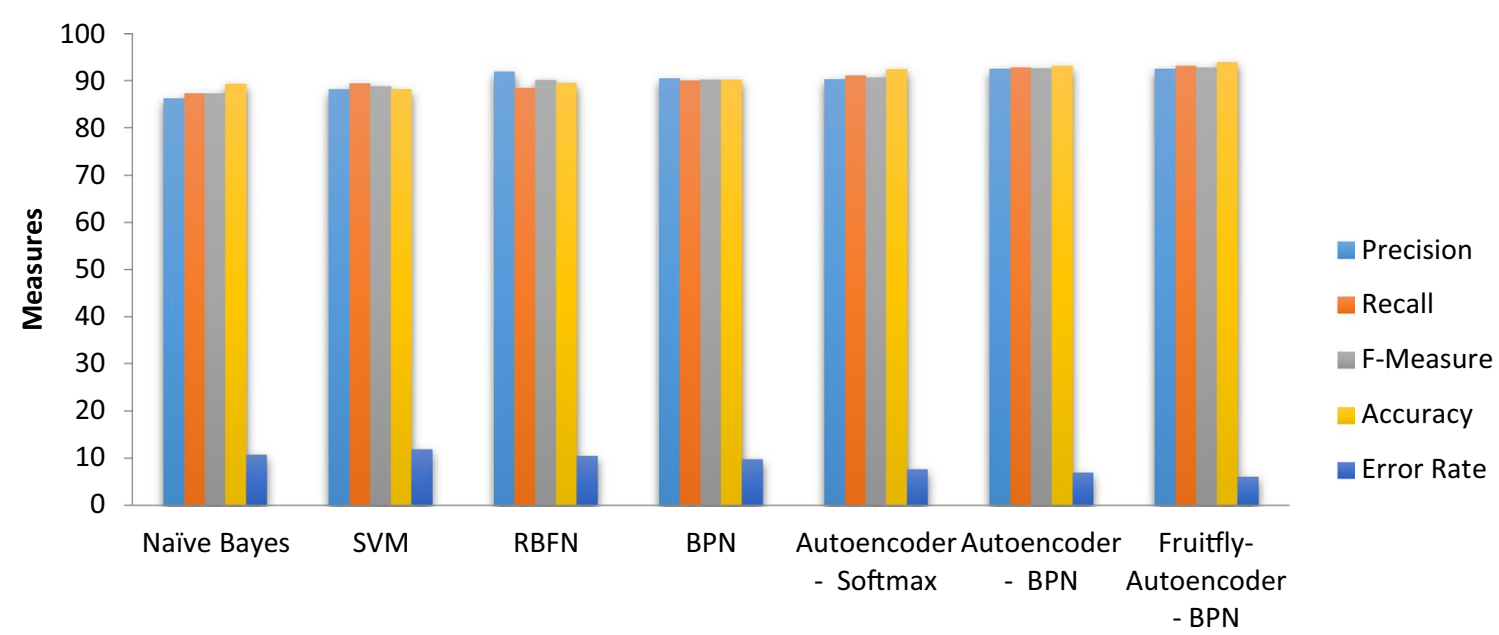

Classifiers

Fig. 7 Relative quantitative measures for intrusion detection system on UNSW-NB15 dataset 
Algorithm 3: Intrusion Detection using Hybridization of Deep Autoencoder with Fruitfly Optimization algorithm

Step 1: Initialize the Fruitfly parameters randomly (population size, position)

Step 2: Train Deep Autoencoder with random hidden values and compute the accuracy.

Step: 3 Compute the cityblock distance of the Fruitfly from the origin

$$
D_{i}=\sum_{i=1}^{n}\left|x_{i}+y_{i}\right|
$$

Step: 4 Find the smell concentration judgment value

$$
S_{i}=\frac{1}{D_{i}}
$$

Step: 5 Compute the smell concentration of the individual Fruitfly

$$
\text { Smell }_{i}=\text { Fitness }_{(} S_{i}
$$

Step: 6 Identify the position of the best smell concentration

$$
\text { [bestSmell_Index] }=\max \left(\text { Smell }_{i}\right)
$$

Step: 7: Go to step 3 until convergence

Step: 8 Train Deep Autoencoder with optimized hidden neurons and find the hidden representation through a deterministic mapping,

$$
\text { Encode_1 }=\sigma_{T}(W * I+b)
$$

Step 9: The latent representation from step 8 is passed as an input to the successive autoencoder,

$$
\text { Encode_2 }=\sigma_{\mathrm{T}}(\mathrm{W} * \text { Encode_1 }+\mathrm{b})
$$

Step 10: Train Back Propagation Neural network with the encoded features to classify the attacks

Step 11: Evaluate the performance of the hybridized deep network 


\subsection{BPN for classification}

In the domain of artificial intelligence, neural networks are promising avenue of research to obtain the complex representations between input and target output [33-37].

The back propagation learning is widely implemented to converge the error. Figure 5 shows the architecture of the BPN network for classification of the attacks in a network where $X_{1}$ to $X_{n}$ indicates the features obtained by Deep Autoencoder from raw data. And $Y_{1}$ to $Y_{5}$ represents class labels of the attack.

\section{Experimental results and discussion}

The proposed IDS using Deep Autoencoder with Fruitfly Optimization has been implemented and discussed. The features obtained from Deep Autoencoder are utilized to classify the attacks of a network system in a better way.

\subsection{Dataset}

The experiments have been conducted on the NSL_KDD and UNSW-NB15 dataset. The NSL_KDD dataset contains 60,741 records with 41 predictor variables and 1 response variable [38]. The UNSW-NB15 dataset contains 1,75,341 records with 42 predictor variables and 1 response variable [39]. The service attribute of UNSW-NB15 dataset contains missing values for 94,205 objects. The objects could be classified as Denial of Service (DoS), User-to-Root (U2R), Remote-to-Locals (R2L), Probe, and Normal in NSL_KDD; Attack and Normal in UNSW-NB15 dataset.

\subsection{Experimental results}

The specifications of BOSTON X86-GPU Rack Server utilized to implement the proposed model is presented in Table 1.

\subsection{Missing value imputation}

For experimentation, five missing combinations have been used with UNSW-NB15 dataset. Further, Root Mean Square (RMSE) and Mean Absolute Error (MAE) are employed as quantitative measures of imputation algorithms.

The comparison between actual and imputed value with various imputation algorithms on UNSW-NB15 dataset is presented in Table 2 . The position $(1340,3)$ contains actual value of 0 . For experimentation, it has been removed and treated as missing value in the dataset. The value obtained with RKMC and FCMRP at $(1340,3)$ are 1 and 0 respectively. Additionally, the value imputed by
FCMRP is closer to the original value in the dataset for all combinations of missing values.

The computational results of RKMC and FCMRP on UNSW-NB15 are given in Table 3. The Root Mean Square Error of RKMC with missing combination of $5 \%, 10 \%$, $15 \%, 20 \%$ and $25 \%$ are $7.1038,5.0140,4.4368,3.4156$, and 3.4039 respectively. The Root Mean Square Error of FCMRP with missing combination of $5 \%, 10 \%, 15 \%, 20 \%$ and $25 \%$ are $4.5378,3.0600,4.1094,2.7115$, and 2.1898 respectively. Further, the quantitative results of cluster analysis with RKMC and FCMRP on UNSW-NB15 are presented in Tables 4 and 5 respectively. The computational results of the FCMRP have been improved as it handles the imprecision in datasets with the exploit of fuzzy and rough sets while preserving crucial information.

\subsubsection{Classifying attacks}

The classification results of the proposed intrusion detection system is compared with various classifiers viz Naïve Bayes, SVM, RBFN, conventional BPN, autoencoder with softmax and autoencoder with BPN. The parameters of Fruitfly algorithm are reported in Table 6 .

\subsubsection{NSL_KDD dataset}

The computational results of the proposed intrusion detection system on NSL_KDD dataset have been discussed. The confusion matrix for attack classification using autoencoder with softmax classifier, autoencoder with backpropagation neural network, and Fruitfly-Autoencoder with back propagation neural network is shown in Tables 7, 8 and 9 respectively.

The quantitative results of the Fruitfly-Autoencoder with BPN for intrusion detection on NSL_KDD dataset have been compared with standard pattern recognition algorithms and are given in Table 10.

From Table 10 and Fig. 6 , it is clearly understood that the Fruitfly-Autoencoder with BPN outperforms the existing classifiers in attack classification. The quantitative results of precision, recall, and F-measure are specified as an average of precision, recall, and F-measure for all classes. The highest and lowest accuracies are $94.00 \%$ for Fruitfly-Autoencoder with BPN and 71.14\% for Naïve Bayes classifier. The classifiers such as Naïve Bayes, SVM, RBFN, BPN, Autoencoder with Softmax, Autoencoder with BPN, and Fruitfly- Autoencoder with BPN provides precision of $70.10 \%, 87.00 \%, 87.70 \%, 89.35 \%, 90.05 \%, 91.35 \%$, and $92.90 \%$ respectively. 


\subsubsection{UNSW-NB15 dataset}

The computational results of the proposed intrusion detection system on UNSW-NB15 dataset have been discussed. The confusion matrix for attack classification using autoencoder with softmax classifier, autoencoder with backpropagation neural network, and Fruitfly-Autoencoder with backpropagation neural network is shown in Tables 11,12 and 13 respectively. The quantitative results of the Fruitfly-Autoencoder with BPN for intrusion detection on UNSW-NB15 dataset have been compared with standard pattern recognition algorithms and are given in Table 14.

From Table 14 and Fig. 7, it is clearly understood that the Fruitfly-Autoencoder with BPN outperforms the existing classifiers in attack classification. The quantitative results of precision, recall, and F-measure are specified as an average of precision, recall, and F-measure for all classes.

The highest and lowest accuracies are $94.00 \%$ for Fruitfly-Autoencoder with BPN and $89.31 \%$ for Naïve Bayes classifier. The classifiers such as Naïve Bayes, SVM, RBFN, BPN, Autoencoder with Softmax, Autoencoder with BPN, and Fruitfly-Autoencoder with BPN provides precision of $86.20 \%, 88.20 \%, 92.00 \%, 90.50 \%, 90.35 \%, 92.50 \%$, and $92.50 \%$ respectively.

The classifiers such as Naïve Bayes, SVM, RBFN, BPN, Autoencoder with Softmax, Autoencoder with BPN, and Fruitfly-Autoencoder with BPN provides F-Measure of $87.30 \%, 88.85 \%, 90.19 \%, 90.30 \%, 90.74 \%, 92.65 \%$, and 92.84\% respectively. Similarly, the Error Rate of Naïve Bayes, SVM, RBFN, BPN, Autoencoder with Softmax, Autoencoder with BPN, and Fruitfly-Autoencoder with BPN are $10.69 \%, 11.80 \%, 10.40 \%, 09.70 \%, 07.60 \%, 06.90 \%$, and $06.00 \%$ respectively. In the proposed model, features extracted from deep Autoencoder with multiple hidden layers are more robust than hand designed feature extractors. In summary, the proposed Fruitfly-Autoencoder with BPN exhibits the highest accuracy of $94.00 \%$ and lowest error rate of $6.00 \%$ than Naïve Bayes, SVM, RBFN, BPN, Autoencoder with Softmax, and Autoencoder with BPN.

\section{Conclusion and future enhancement}

Intrusion Detection can be considered as more vital to safeguard the crucial information in a network system of an organization. More recently, deep learning approach is widely employed in many real world problems to achieve better prediction. Hence, in this research work, a hybridized method using Deep Autoencoder with Fruitfly Optimization is introduced for classifying the attacks. Initially, missing values in the dataset have been imputed with the Fuzzy C-Means Rough Parameter method. Then, the more discriminate features are extracted from the raw dataset with the exploit of Deep Autoencoder with more hidden layers. Furthermore, the hidden neurons of Deep Autoencoder are optimized with the meta-heuristic Fruitfly Optimization algorithm. Finally, BPN has been utilized to predict the attacks. The proposed Fruitfly-Autoencoder with BPN produced high accuracy than benchmark classifiers such as Naïve Bayes, SVM, RBFN, BPN, Autoencoder with Softmax, and Autoencoder with BPN. To validate the proposed IDS, several experiments have been conducted on NSL_KDD and UNSW-NB15 dataset. The proposed model was executed on GPU version P100 as they process multiple computations in parallel. The acquired results expound that the proposed method reveals better results in terms of precision, recall, f-measure, accuracy and error rate. In future, other Deep Learning models will be integrated to classify attacks in the network system. Further, the proposed approach could be enhanced by incorporating latest optimization algorithms such as cooperative coevolutionary differential evolution, whale optimization, and Biogeography-based Optimization.

Acknowledgements Author's would like to thank UGC, New Delhi for the financial support received under UGC SAP DRS [F. 5-6/2018/ DRS-II (SAP-II)].

Declaration

Conflict of interest The authors declare that they have no conflicts of interest.

Open Access This article is licensed under a Creative Commons Attribution 4.0 International License, which permits use, sharing, adaptation, distribution and reproduction in any medium or format, as long as you give appropriate credit to the original author(s) and the source, provide a link to the Creative Commons licence, and indicate if changes were made. The images or other third party material in this article are included in the article's Creative Commons licence, unless indicated otherwise in a credit line to the material. If material is not included in the article's Creative Commons licence and your intended use is not permitted by statutory regulation or exceeds the permitted use, you will need to obtain permission directly from the copyright holder. To view a copy of this licence, visit http://creativecommons. org/licenses/by/4.0/.

\section{References}

1. Liao HJ, Lin CHR, Lin YC, Tung KY (2013) Intrusion detection system: a comprehensive review. J Netw Comput Appl 36(1):16-24

2. Lazarevic A, Kumar V, Srivastava J (2005) Intrusion detection: asurvey. In: Managing cyber threats (pp 19-78). Springer, Boston, $\mathrm{MA}$

3. Mukherjee B, Heberlein LT, Levitt KN (1994) Network intrusion detection. IEEE Network 8(3):26-41

4. Elsherif $A$ (2018) Automatic intrusion detection system using deep recurrent neural network paradigm. J Inf Secur Cybern Res 1(1):28-41 
5. Depren O, Topallar M, Anarim E, Ciliz MK (2005) An intelligent intrusion detection system (IDS) for anomaly and misuse detection in computer networks. Expert Syst Appl 29(4):713-722

6. Schmidhuber J (2015) Deep learning in neural networks: an overview. Neural Netw 61:85-117

7. Deng Y, Ren Z, Kong Y, Bao F, Dai Q (2016) A hierarchical fused fuzzy deep neural network for data classification. IEEE Trans Fuzzy Syst 25(4):1006-1012

8. Lin SM (2013) Analysis of service satisfaction in web auction logistics service using a combination of fruit fly optimization algorithm and general regression neural network. Neural Comput Appl 22(3):783-791

9. Tang J, Zhang G, Wang Y, Wang H, Liu F (2015) A hybrid approach to integrate fuzzy C-means based imputation method with genetic algorithm for missing traffic volume data estimation. Transp Res Part C Emerg Technol 51:29-40

10. Tian J, Gao M, Zhang F (2009) Network intrusion detection method based on radial basis function neural network. In: IEEE, 978-1-4244-4589-9/\$25@2009

11. Norouzian MR, Merati S (2011) Classifying attacks in a network intrusion detection system based on artificial neural networks. In: ICACT 2011, ISBN 978-89-5519-155-4, Feb 13-16, 2011

12. Xiangmei L, Zhi Q (2011) The application of hybrid neural network algorithms in intrusion detection system. IEEE, 978-1-4244-8694-6/11/\$26@2011

13. Yin C, Zhu Y, Fei J, He X (2017) A deep learning approach for intrusion detection using recurrent neural networks. IEEE Access 5:21954-21961

14. Alzubi QM, Anbar M, Alqattan ZN, Al-Betar MA, Abdullah R Intrusion detection system based on a modified binary grey wolf optimisation. Neural Comput Appl 1-13

15. Qureshi AS, Khan A, Shamim N, Durad MH Intrusion detection using deep sparse auto-encoder and self-taught learning. Neural Comput Appl 1-13

16. Aslahi-Shahri BM, Rahmani R, Chizari M, Maralani A, Eslami M, Golkar MJ, Ebrahimi A (2016) A hybrid method consisting of GA and SVM for intrusion detection system. Neural Comput Appl 27(6):1669-1676

17. Rekha G, Malik S, Tyagi AK, Nair MM (2020) Intrusion detection in cyber security: role of machine learning and data mining in cyber security. Adv Sci Technol Eng Syst J 5(3):72-81

18. Drewek-Ossowicka A, Pietrołaj M, Rumiński J (2020) A survey of neural networks usage for intrusion detection systems. J Ambient Intell Hum Comput 1-18

19. Liu G, Zhang J (2020) CNID: research of network intrusion detection based on convolutional neural network. Discrete Dynamics in Nature and Society

20. Raja PS, Thangavel K (2020) Missing value imputation using unsupervised machine learning techniques. Soft Comput 24(6):4361-4392

21. Raja PS, Sasirekha K, Thangavel K (2019) A novel fuzzy rough clustering parameter-based missing value imputation. Neural Comput Appl 1-18

22. Alain G, Bengio Y (2012) What regularized auto-encoders learn from the data generating distribution. Technical Report
23. Chen M, Xu Z, Winberger K, Sha F (2012) Marginalized denoising autoencoders for domain adaptation. ICML

24. Swersky K, Ranzato M, Buchman D, Marlin B, de Freitas N (2011) On score matching for energy based models: generalizing autoencoders and simplifying deep learning. In: International conference on machine learning ICML-11, ( $p$ 1201)

25. Sasirekha K, Thangavel K (2020) A novel biometric image enhancement approach with the hybridization of undecimated wavelet transform and deep autoencoder. In: Handbook of research on machine and deep learning applications for cyber security (pp 245-269). IGI Global

26. Vincent $P$ (2011) A connection between score matching and denoising autoencoders. Neural Comput 23(7):1661-1674

27. Vincent $P$, Larochelle $H$, Bengio $Y$, Manzagol PA (2008) Extracting and composing robust features with denoising autoencoders. In: ICML

28. Rifai S, Vincent P, Muller X, Glorot X, Bengio Y (2011) Contractive auto-encoders: explicit invariance during feature extraction. In: Proceedings of ICML (pp 833-840)

29. Yu J, Zheng X, Wang S (2019) A deep autoencoder feature learning method for process pattern recognition. J Process Control 79:1-15

30. Pan WT (2012) A new Fruitfly Optimization algorithm: taking the financial distress model as an example. Knowl-Based Syst 26:69-74

31. Pan QK, Sang HY, Duan JH, Gao L (2014) An improved Fruitfly optimization algorithm for continuous function optimization problems. Knowl-Based Syst 62:69-83

32. Li HZ, Guo S, Li CJ, Sun JQ (2013) A hybrid annual power load forecasting model based on generalized regression neural network with Fruitfly optimization algorithm. Knowl-Based Syst 37:378-387

33. Sasirekha K, Thangavel K (2018) A novel fingerprint classification system using BPNN with local binary pattern and weighted PCA. Int J Biometr 10(1):77-104

34. Linda O, Vollmer T, Manic M (2009) Neural network based intrusion detection system for critical infrastructures. In: 2009 international joint conference on neural networks (pp 1827-1834). IEEE

35. Moradi M, Zulkernine M (2004) A neural network based system for intrusion detection and classification of attacks. In: Proceedings of the IEEE international conference on advances in intelligent systems-theory and applications (pp 15-18)

36. Lippmann RP, Cunningham RK (2000) Improving intrusion detection performance using keyword selection and neural networks. Comput Netw 34(4):597-603

37. Zhang C, Jiang J, Kamel M (2005) Intrusion detection using hierarchical neural networks. Pattern Recognit Lett 26(6):779-791

38. NSL_KDD dataset: https://www.unb.ca/ cic/datasets

39. UNSW-NB15,dataset: https://www.unsw.adfa.edu.au/unswcanberra-cyber/ cybersecurity/ ADFA-NB15-Datasets

Publisher's Note Springer Nature remains neutral with regard to jurisdictional claims in published maps and institutional affiliations. 